\title{
Fault Diagnosis Based on EEMD-KPCA-MTS for Rolling Bearing
}

\section{Weiyu HAN and Longsheng CHENG}

School of Economics and Management, Nanjing University of Science and Technology, P.R. China hwy94nj@163.com

Keywords: fault diagnosis, KPCA, MTS, EEMD, IMF

Abstract. To improve the accuracy of fault diagnosis for rolling bearing, an integrated fault diagnosis method based on EEMD (Ensemble Empirical Mode Decomposition), KPCA (Kernel Principal Component Analysis) and MTS (Mahalanobis Taguchi System) is proposed. Firstly, EEMD decompose the non-stationary and nonlinear vibration signals into a series of IMFs (intrinsic mode functions). Then some of IMFs sensitive to the fault information is selected by the IMF sensitive discriminant algorithm and establish the initial feature vector. Then, KPCA further reduces the dimensionality of the vector. Finally, an effective MTS fault detectors and classifiers is established to identify fault types of sample data. The experimental results show that compared with conventional single fault diagnosis methods, the EEMD-KPCA-MTS model has strong adaptability and accuracy.

\section{Introduction}

As a core component of rotating machinery, rolling bearings play an important role in the operation of mechanical equipment [1]. $40 \%$ mechanical equipment failures are related to bearings [2]. Therefore, it has important practical significance for the fault diagnosis of rolling bearings.

Signal feature extraction is the key technology for fault diagnosis of rolling bearings. EEMD is an effective method for dealing with non-stationary and nonlinear signals, which avoid the mode aliasing phenomenon caused by EMD [3-4]. However, only part of the IMFs decomposed by EEMD can reflect the fault characteristics, while others are interference components. The accuracy of the model is affected if the interference components are not eliminated [5]. Many scholars combine EEMD with neural network, support vector machine and other pattern recognition algorithms for fault identification. These methods rely on the existing knowledge in the knowledge base [6-8]. When a new abnormal situation appears, it may be not available. Therefore, how to find an emerging pattern recognition method is an important direction of rolling bearings.

To solve the above problem, an integrated fault diagnosis method based on EEMD, KPCA and MTS is proposed. Firstly, EEMD decompose the vibration signals into a series of IMFs, and then some of IMFs sensitive to the fault information is selected by the IMF sensitive discriminant algorithm and establish the initial feature vector. Then, KPCA further reduces the dimensionality of the vector. Finally, use MTS to identify fault types of sample data.

\section{EEMD Method and Correlation-Based IMF Sensitive Discriminant Algorithm}

The EEMD method is improved on the basis of EMD. EMD decompose original signal into a series of IMFs. Since the vibration signal is collected during the operation of the bearing, the noise component is large. So the signal needs to be denoised. However, it should be emphasized that it is necessary to accurately select the critical point to remove Gaussian white noise using EMD, otherwise it may cause serious distortion of the signal. EEMD adds white noise to the signal, so that the signal is continuous at different scales which causes the decomposition effect better. At the same time, according to the characteristic of zero mean of Gauss white noise, the white noise is added to the signal repeatedly. After averaging, the noise is offset, and the more frequency, the less effect of the noise.

After the EEMD decomposition of the signal, only part of the IMF component is sensitive to the fault information, and other IMF components are the interference components. Therefore, to ensure the accuracy of the analysis, an IMF sensitive discriminant algorithm based on mode function 
correlation evaluation is used to select the IMF component sensitive to the fault information. The specific steps of the algorithm are as follows:

1) Calculate the correlation coefficient $p_{i}$ between the fault signal $\mathrm{x}(\mathrm{t})$ and its various IMF components $c_{1}(t), c_{2}(t), \ldots, c_{n}(t)$

$$
\rho_{i}=\sum_{t=0}^{\infty} \sum_{k=i}^{n} c_{k}(t) x(t) / \sqrt{\sum_{t=0}^{\infty} \sum_{k=i}^{n} c_{k}^{2}(t) x^{2}(t)}
$$

2) Calculate the sensitivity factor of each IMF component,

$$
\lambda_{i}=\frac{\rho_{i}-\min (\rho)}{\max (\rho)-\min (\rho)}(i=1,2, \ldots n)
$$

3) Reorder IMFs by sensitivity factor, and get a new IMF sequence $\left\{c_{n}^{\prime}\right\}(i=1,2, \ldots n)$, and calculate the difference between two adjacent sensitivity coefficients:

$$
d_{n}=\lambda_{n}^{\prime}-\lambda_{n-1}^{\prime}
$$

4) Determine the serial number $\mathrm{k}$ corresponding to the maximum value of the sensitivity coefficient difference, then the first $k$-th order IMF in $\left\{c_{n}\right\}$ is the fault-sensitive IMF. The algorithm considers the similarity between the IMF and the original signal, and aims to highlight the components related to the fault characteristics and weaken the influence of the unrelated components.

\section{Mahalanobis Taguchi System and MTS Fault Detection and Classification Model}

As a multi-dimensional pattern recognition method, the main purpose of the MTS is to measure the abnormality of the sample and the degree of abnormality by constructing measurement scales [9-10]. MTS combines the MD and the Taguchi method. MTS use the MD as a reference to calculate the distance of the sample. The Taguchi method includes an orthogonal table and a signal-to-noise ratio. The orthogonal table and the signal-to-noise ratio are used together as a tool for screening feature variables, thereby reducing the dimensionality. However, the actual results show that the Taguchi method is not ideal for the dimension reduction of the IMF component. Therefore, this paper uses the KPCA method instead of the Taguchi method. MTS fault detection and classification model are shown as follow:

Step one, construct the reference space of normal sample.

Assume that there are one normal type and $\mathrm{L}$ fault types, each type collects $\mathrm{m}$ sample data. So there are $m$ normal sample data and $\mathrm{p}$ abnormal sample data. After EEMD decomposition and the selection of IMF sensitive discriminant algorithm, an initial feature vector is constructed by calculating the parameters of time domain statistics. Assume that extract a total of $\mathrm{n}$ feature vectors, and $\mathrm{m}$ normal sample data construct $m \times n$ sample space. Where $a_{i j}$ is the value of the $i$-th sample in the $j$-th component.

1) Calculate the mean and standard deviation of each feature vector in the normal sample data matrix A,

$$
u_{j}=\frac{1}{m} \sum_{i=1}^{m} a_{i j}, S_{j}=\sqrt{\frac{1}{m-1} \sum_{i=1}^{m}\left[a_{i j}-u_{j}\right]^{2}}, j=1,2 \ldots, n
$$

2) Use $u_{j}$ and $s_{j}$ to standardize the sample data matrix A

$$
Z_{i j}=\frac{a_{i j}-u_{j}}{s_{j}}, i=1,2, \ldots, m, j=1,2, \ldots, n
$$


get normalized normal sample data matrix $Z=\left[\begin{array}{cccc}z_{11} & z_{12} & \mathrm{~L} & z_{1 n} \\ z_{21} & z_{22} & \ldots & z_{2 n} \\ \mathrm{M} & \mathrm{M} & \mathrm{M} & \mathrm{M} \\ z_{m 1} & z_{m 2} & z_{m 3} & z_{m n}\end{array}\right]$

3) Calculate the correlation coefficient matrix between the attributes,

$$
S=\frac{1}{m-1} \sum_{i=1}^{m} Z_{i}^{T} Z_{i}
$$

In the formula, $Z_{i}=\left[z_{i 1}, z_{i 2}, \mathrm{~L}, z_{i m}\right]$ is the $i$-th normalized sample data of matrix $Z$.

4) Calculate the inverse matrix $S^{-1}$ of the correlation coefficient matrix $S$ between the feature vectors.

5) Calculate the MD of all normal samples

$$
M D_{i}^{2}=\frac{1}{n} Z_{i} S^{-1} Z_{i}^{T}, i=1,2, \mathrm{~L}, m
$$

Step two, confirm the validity of the reference space.

1)Treat all fault samples as negative samples and $\mathrm{p}$ negative sample data form $p \times n$ matrix $B$.Standardize the abnormal fault sample data matrix $B$ using $u_{j}$ and $s_{j}$ calculated in step one,

$$
v_{k j}=\frac{b_{k j}-u_{j}}{s_{j}}, k=1,2, \mathrm{~L}, p, j=1,2, \mathrm{~L}, n
$$

get normalized normal sample data matrix $V=\left[\begin{array}{cccc}v_{11} & v_{12} & \mathrm{~K} & v_{1 n} \\ v_{21} & v_{22} & \mathrm{~L} & v_{2 n} \\ \mathrm{M} & \mathrm{M} & \mathrm{M} & \mathrm{M} \\ v_{p 1} & v_{p 2} & \mathrm{~L} & v_{p n}\end{array}\right]$

2) Calculate the MD of all abnormal fault samples

$$
M D_{k}^{2}=\frac{1}{n} V_{k} S^{-1} V_{k}^{T}, k=1,2, \mathrm{~L}, p
$$

The greater the difference of MD between the abnormal fault sample and the normal sample, the more effective the reference space is.

Step three, Construct L fault types Mahalanobis space.

1) Build L fault types reference space, repeat step one.

Calculate the mean and standard deviation vectors, the correlation coefficient matrix, and the MD of each type of fault sample separately. Construct Mahalanobis spaces for each fault mode samples.

2) Verify the validity of L reference space, repeat step two

Regard a certain type of fault sample as positive samples, and other types of sample data are regarded as negative samples. Verify the validity of positive sample reference space by calculating the MD of negative sample data. If the MD of the negative sample is greater than the MD of the positive sample in every Mahalanobis space, the model measurement scale is good.

\section{Step four, KPCA optimization reference space}

Select the appropriate kernel function and kernel parameters, use KPCA to process the $(m+p) \times n$ matrix, and extract the main components of the matrix and compress the matrix to optimize the Mahalanobis space. Set the cumulative contribution rate of compression to greater than $95 \%$ and compress $\mathrm{n}$ parameters to $\mathrm{t}$. Thereby a $(m+p) \times t$ matrix is obtained.

\section{Step five, Predict the sample}

Firstly, calculate the MD of the sample in the Mahalanobis space of normal sample, and detect if the sample is normal. Then, calculate the MD of the sample in each fault Mahalanobis space and the 
sample is classified into a certain fault mode with strict criteria. The next chapter will explain in detail.

\section{Experimental Analysis}

\subsection{Introduction to experimental data}

The experimental research was carried out by using the vibration data of the bearing fault test bench of Case Western Reserve University. Rolling bearings have four states, normal, inner ring fault, rolling fault and outer ring fault. Analyze the one end (Driver-D) vibration signal collected by the vibration sensor. Each sample is a signal composed of 1024 sampling points. The normal signal takes 100 samples, and each of the three fault signals takes 100 samples, a total of 400 samples. Among them, 80 samples of each type signal are randomly selected as the training set, and the remaining 20 samples are used as test sets. The vibration image of the original signal is shown in Figure 1.

\subsection{EEMD decomposition of vibration signal}

Use EEMD to decompose the original vibration signal, and the IMF components are obtained, and the signal decomposition of one of the samples is shown in Figure 2. The signal is the original signal, IMF1, IMF2, ... IMF9 is the characteristic component of each feature, RES is the remainder, the horizontal axis represents the sampling point, and the vertical axis represents the acceleration amplitude.

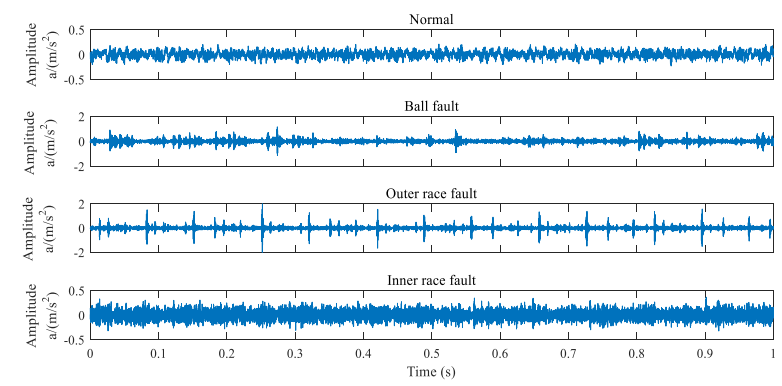

Fig.1 The waveforms of rolling bearing vibration under four different conditions signal.

\subsection{Select sensitive IMF components}

Use IMF sensitive discriminant algorithm to select the IMF component sensitive to the fault information. Calculate the correlation coefficient between the IMF component and the original signal in each state. Figure 3 shows IMF correlation coefficient of normal samples. It can be seen from the figure that IMF1-6 is selected as the sensitive IMF component under normal conditions.

The results of selecting the IMF component for each state are shown in Table 1. Finally, IMF1-6 components were selected as the feature set source.

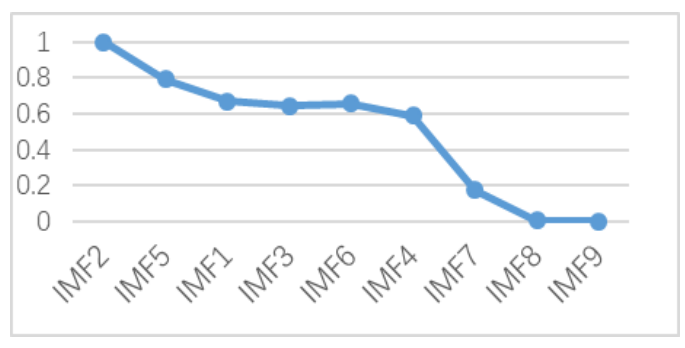

Fig.3 IMF correlation coefficient of Normal sample.
Table 1. The sensitive IMF results of fault signals.

\begin{tabular}{cc}
\hline Type & Sensitive IMFs \\
\hline Normal & IMF1, IMF2, IMF3, IMF4, IMF5, IMF6 \\
Rolling fault & IMF1 \\
Inner ring fault & IMF1 \\
Outer ring fault & IMF1, IMF3
\end{tabular}




\subsection{Build multiple Mahalanobis}

Based on the IMF1-IMF6 component selected in this paper, the corresponding four statistical eigenvalues are calculated separately, which are rms, range, energy, and kurtosis. That means a 24-dimensional eigenvector is extracted from each sample and form four $100 \times 24$ feature spaces. Then, according to steps 1 to 3 of the previous chapter, the Mahalanobis space of normal sample and three fault types sample are constructed separately.

\subsection{KPCA optimizes Mahalanobis spaces}

First, organize the signals decomposed by EEMD into fault data sets. In this system, there are 24 characteristic variables. And then use KPCA to reduce the dimension of the $400 \times 24$ data set composed by the 100 samples data of normal, inner ring fault, outer ring fault, and rolling fault signal. In this paper, RBF radial basis kernel function is used for processing. After KPCA calculation, the data matrix arranged in descending order of correlation is obtained. According to the cumulative contribution rate of principal component, the principal component is more than $95 \%$, and 7 principal components are taken respectively to obtain a new $400 \times 7$ dimensional data, which is used as a sample for subsequent classification processing. The Figure 4 shows cumulative contribution rate of principal components.

\subsection{Construct new Mahalanobis space and set threshold}

Then 80 samples of each type of signal are randomly selected as the training set, and the remaining 20 samples are used as test sets. Use the dimensionality-reduced feature space $(320 \times 7)$ to construct a new Mahalanobis space. Then, according to steps 1 to 3 of the previous chapter, the mean, standard deviation and correlation matrix between the initial characteristic variables of each fault type are calculated, and these data are composed into the reference space. All other fault data and normal data are selected as negative samples. For example, take normal sample as a positive sample, by calculating the MD obtained from the normal data (see Figure 5), it is found that the positive sample space is mostly near 1 , and the MD of the negative sample space is significantly different.

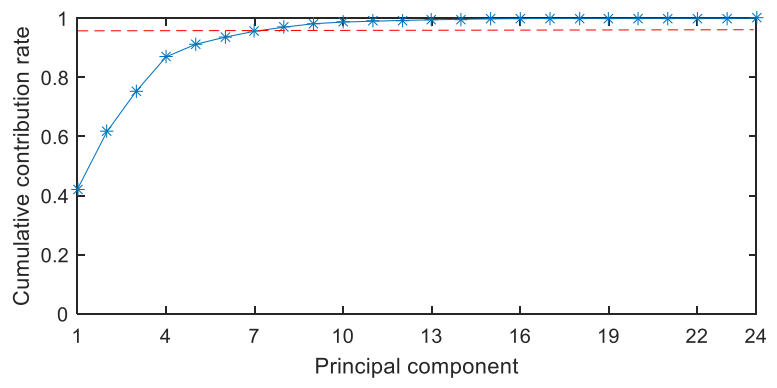

Fig. 4 Cumulative contribution rate of KPCA.
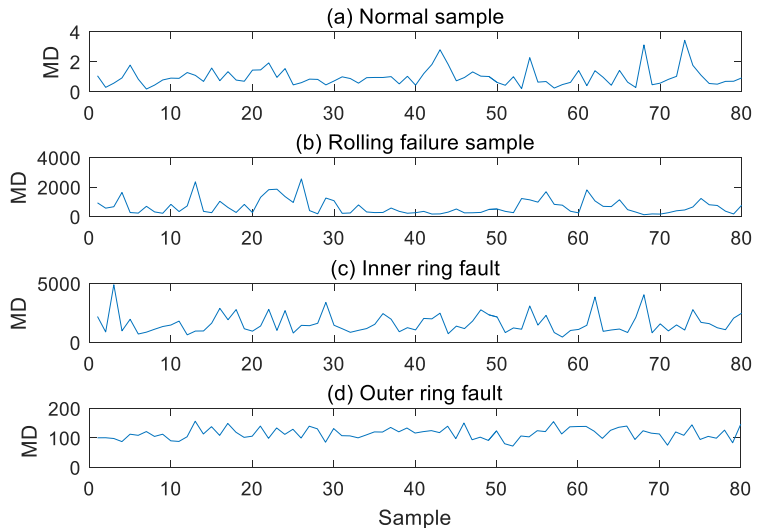

Fig. 5 The MD in different types.

And then set a threshold for each Mahalanobis space. This paper uses $3 \sigma$ principles to determine the threshold. First, the fault detection is performed to determine whether the simple has faulted. By calculating, the threshold of the Mahalanobis space of the normal sample is 2.78 . That is to see, the $M D$ of the sample to be judged in the normal Mahalanobis space is calculated as $M D_{0}$. If $M D_{0}<$ 2.78, it is judged to be normal type. If $M D_{0}>2.78$, it is judged to be a fault class.

Then, the thresholds of the inner ring fault, outer ring fault and rolling element fault are 3.41, 3.29, 2.52 respectively. For an abnormal sample, calculate its MD in three Mahalanobis spaces, denoted as $M D_{1}, M D_{2}, M D_{3}$, and the judgment rule is:

1) If $M D_{1}<3.41, M D_{2}>3.29, M D_{3}>2.52$, then the sample is classified as the inner ring fault.

2) If $M D_{2}<3.29, M D_{1}>3.41, M D_{3}>2.52$, then the sample is classified as the outer ring fault. 
3) If $M D_{3}<2.52, M D_{1}>3.41, M D_{2}>3.29$, then the sample is classified as the rolling fault.

4) If $M D_{1}, M D_{2}$, and $M D_{3}$ have two or three values that are not greater than the corresponding threshold, the sample is classified as the class corresponding to the minimum $M D$.

5) If three values in $M D_{1}, M D_{2}$, and $M D_{3}$ are greater than the corresponding threshold, the sample is considered normal.

\subsection{Results Analysis}

Retrieve the training data and then applied to the test data set. Overall, the accuracy of the model is $93 \%$. Among them, the fault detector works best, reaching $100 \%$. The accuracy of the fault classifier is $88 \%$. This result is consistent with the actual situation, because the normal class signal is the most different from other fault signals. The result shows the model proposed in this paper is effective.

\section{Conclusion}

This paper proposes a fault detection and classification method for rolling bearings based on EEMD-KPCA-MTS. First, this method uses the advantages of EEMD to solve the pattern mixing problem. And the IMF algorithm can effectively eliminate the IMF that is not related to the fault information, increasing the accuracy of feature extraction. Then, Use KPCA to extract nonlinear features of fault data, reduce computational cost and improve calculation accuracy. Finally, this method effectively diagnose and classify faults by calculating the Mahalanobis distance of sample and the threshold of Mahalanobis space. The method proposed in this paper can eliminate the interference components and highlight the fault characteristics, thus perfecting the theoretical system of the MTS and providing a new solution for the multi-fault classification method. The results of the case analysis verify the effectiveness of the proposed method, which provides a feasible way for fault diagnosis of rolling bearings

\section{References}

[1] Tribeni Prasad Banerjee, Swagatam Das. Multi-sensor data fusion using support vector machine for motor fault detection [J]. Information Sciences, 2012, 217.

[2] Hojat Heidari Bafroui,Abdolreza Ohadi. Application of wavelet energy and Shannon entropy for feature extraction in gearbox fault detection under varying speed conditions [J]. Neurocomputing, 2014,133.

[3] Wang Y S,Ma Q H,Zhu Q,et al.An intelligenct approach for engine fault diagnosis based on Hilbert-Huang transform and support vector machine[J].Applied Acousties,2014(75):1-9

[4] Shen Yin,Steven X. Ding,Adel Haghani,Haiyang Hao,Ping Zhang. A comparison study of basic data-driven fault diagnosis and process monitoring methods on the benchmark Tennessee Eastman process [J]. Journal of Process Control, 2012, 22 (9).

[5] Xu Yuan,liu Ying,Zhu qunxiong. A complex process fault prognosis approach based on multivariate delayed sequences[J]. CIESC Journal,2013,64(12):4290-4295

[6] Jaouher Ben Ali,Nader Fnaiech,Lotfi Saidi,Brigitte Chebel-Morello,Farhat Fnaiech. Application of empirical mode decomposition and artificial neural network for automatic bearing fault diagnosis based on vibration signals [J]. Applied Acoustics, 2015, 89.

[7] Wu Bin,YU Shanping,LUO Yuegang,et al.Rolling bearing faults diagnosis method based on SVM-HMM[J].Measuring Technology and Mechatronics Automation,2010,3:295-298

[8] AO H L, CHENG J LI K,et al.A roller bearing fault diagnosis method based on LCD energy rntropy and ACROA-SVM[J].Shock and vibration,2014. 
[9] Changqing Shen,Dong Wang,Fanrang Kong,Peter W. Tse. Fault diagnosis of rotating machinery based on the statistical parameters of wavelet packet paving and a generic support vector regressive classifier [J]. Measurement, 2013, 46(4).

[10] AO H L, CHENG J LI K,et al.A roller bearing fault diagnosis method based on LCD energy rntropy and ACROA-SVM[J].Shock and vibration,2014. 